\title{
Relationship Between Insulin Resistance and Serum Androgens Levels in Women With and Without PCOS by Rotterdam Diagnosis Criteria
}

\author{
Maryam Movahedinejad \\ Master's College: The Master's University \\ Saeide Ziaei \\ Master's College: The Master's University \\ Anooshiravan Kazemnezhad \\ Master's College: The Master's University \\ zahra kamali ( $\nabla$ kamalishahla6@gmail.com ) \\ Master's College: The Master's University
}

\section{Research}

Keywords: Polycystic Ovarian Syndrome, Insulin Resistance, Serum Androgens Level, Rotterdam

Posted Date: September 14th, 2021

DOI: https://doi.org/10.21203/rs.3.rs-864589/v1

License: (c) (i) This work is licensed under a Creative Commons Attribution 4.0 International License. Read Full License 


\section{Abstract}

Introduction

PCOS is the most common endocrine disorder in women of childbearing age. Insulin resistance is a key component of the pathophysiology of PCOS that can increase the secretion of serum androgens. The aim of this study was to determine the relationship between insulin resistance and serum androgens levels in PCOS subgroups.

Methods

Hormonal and clinical laboratory studies were performed for all research units. Insulin resistance was diagnosed with the HOMA index (Cut off $>2.5)$.

Results

In subgroup $A$, there was a positive relationship between HOMA-IR with FAI $(P=0.014)$ and negative relationship with SHBG $(P<0.001)$. In subgroup $C$, a negative relationship was observed between HOMA-IR and SHBG $(P=0.002)$. In subgroup $D$, there was a positive relationship between HOMA-IR and FAI $(P=0.035)$. No significant association was found in the control group and subgroup $B(P>0.05)$. Also, in all different PCOS phenotypes, no association was found between HOMA-IR and serum TT levels $(P>0.05)$.

Conclusion

There was a relationship between insulin resistance and some serum androgens in PCOS subtypes. Therefore, it is hoped that early detection of these at-risk patients will be done through early biochemical markers to prevent and change lifestyle.

\section{Background}

Polycystic ovary syndrome (PCOS) is a heterogeneous endocrine disorder (1). The prevalence of PCOS according to the Rotterdam Diagnostic Criteria (ESHRE) has been reported to vary between $6.5 \%$ and $16 \%$ (2). PCOS is difficult to diagnose due to the heterogeneous nature and possible changes in patient status. At present, the most commonly used criterion for diagnosis is the Rotterdam criterion based on the 2003 agreement (3). Two criteria of the following three criteria are essential for the diagnosis of PCOS: 1. No ovulation or oligovascularization (number of cycles less than 6 cycles over 12 months). 2. Clinical or biochemical signs of increased androgens (including acne, hirsutism, alopecia, or elevated serum levels of androgens) 3. Polycystic view on ultrasound (presence of 12 follicles or more in each ovary with a diameter between 2-9 mm or ovarian volume exceeding $10 \mathrm{~cm} 3$ ) (4). In Polycystic Ovary Syndrome, the pituitary gland releases high levels of the luteinizing hormone and the ovaries produce high levels of androgens. This disrupts the normal menstrual cycle and can lead to infertility, Hair-covered body and acne (5). The exact cause of Polycystic Ovary Syndrome is unclear, but insulin resistance is a fundamental component of the pathophysiology of PCOS (6). Insulin resistance is defined as a defective response to glucose to a certain amount of insulin. In many of these patients, circulating insulin levels are elevated to maintain glucose levels. This condition is associated with a wide range of manifestations including cardiovascular disease, hypertension, type 2 diabetes and lipid disorders (7). These results demonstrate the importance of identifying early biochemical markers to prevent this syndrome (8).

As many as $65-70 \%$ of PCOS women are insulin resistant (9). Today, insulin resistance and hyperinsulinemia are well-known symptoms of polycystic ovary syndrome, which increase the secretion of ovarian androgens and decrease the levels of sex hormone-binding globulin (SHBG) (10). SHBG is the main transport protein for testosterone and estradiol that modulates their biological activity (8). This decrease leads to an increase in free and available testosterone (11). Hyperinsulinemia, on the other hand, disrupts the patient's cytochrome enzyme (P450 17 alpha) in the ovaries and adrenals and increases the secretion of adrenal and ovarian androgens. Therefore, they have a major role in the development and exacerbation of hyperandrogenic symptoms in these patients (12). 
In Ducluzeau's (2003) study, sex hormone binding globulin (SHBG) was associated with IR. But SHBG cannot act as an IR predictor. In this study, both obese and lean women were exposed to insulin resistance (8). In the study of Kajaia (2007), women classified using insulin resistance indices showed significantly higher BMI and FAI and lower levels of SHBG and HDL. However, SHBG may act as a predictive marker of IR in these women, especially in those who are obese (13).

In general, many studies show a high degree of association between hyperandrogenism (HA) and insulin resistance (IR). The association between HA and IR appears to be different between women with PCOS and non-PCOS (14).

According to the above, Polycystic Ovary Syndrome (PCOS) in women has been suggested as the main cause of androgen hormone deficiency. This is associated with a high risk of cardiovascular disease and type 2 diabetes. In many PCOS patients, increased fasting insulin and glucose intolerance are associated with increased insulin resistance, which has been identified as a new target of therapeutic strategy. Identification of at-risk patients through primary biochemical markers will also be useful for prevention and early change in lifestyle (8). On the other hand, no study was found to evaluate the association between hyperandrogenism (HA) and insulin resistance (IR) with different PCOS phenotypes based on Rotterdam criteria in women with and without PCOS. Therefore, the researchers designed and conducted a study with the aim of "Relationship between insulin resistance and serum androgens in patients with PCOS in four subgroups of this disease based on Rotterdam diagnostic criteria".

\section{Methods}

The present study is a case-control study to investigating serum androgen levels in four subgroups of polycystic ovary syndrome and control group with insulin resistance, with the approval of the Medical Ethics Committee of Tarbiat Modares University in Tehran, Iran. 182 participants were recruited during 2016-2017. The sample size based on the results of the pilot study and the correlation between insulin resistance index and androgenic components showed that the minimum correlation between these indices was 0.50 . Therefore, with $95 \%$ confidence and $80 \%$ test power, the number of samples required was 26.2 based on the following formula for each subgroup with approximately 27 considered. Considering $20 \%$ of sample loss, the final volume per phenotype was estimated at 31 individuals.

$$
\begin{aligned}
& n=\frac{\left(z_{1-\frac{\alpha}{2}}+z_{1-\beta}\right)^{2}}{(c(r))^{2}}+3 \\
& C(r)=\frac{1}{2} \log _{e} \frac{1+r}{1-r}=\frac{1}{2} \log _{e} \frac{1+0.5}{1-0.5}=0.55 \\
& n=\frac{(1.96+0.84)^{2}}{(0.55)^{2}}+3=26.2 \cong 27
\end{aligned}
$$

$$
27+0.2(27)=31
$$

The sampling method was available. Subjects were selected from referrals to gynecology ward, endocrinology ward of Arash hospital and selected private clinic if they were eligible to enter our study after filling informed consent. Inclusion criteria in the case group were: Iranian race, age 18-40 years, lack of chronic metabolic and non-metabolic diseases affecting diet such as diabetes mellitus, hyperthyroidism, hypothyroidism, and hyperlipidemia, not taking medicines that affect appetite and diet, no specific diet, no pregnancy, no hormone use for three months before starting the study. In the event of any problems, including pregnancy and illness requiring specific drug use, the research units were excluded. Inclusion criteria in the control group were: 18-40 years old and without any diagnostic criteria for PCOS (non-hirsute: without excessive hair growth, with regular ovulation cycles) referred to Women's Clinic for other reasons. Then the two groups were matched for education, BMI, economic status, and physical activity and exercise status.

Page $3 / 13$ 
Initially, the disease was diagnosed after excluding other abnormalities that mimic the PCOS phenotype (ovarian or adrenal neoplasm, Cushing's syndrome, Hyperprolactinemia, Thyroid Disease and Congenital Adrenal Hyperplasia Starting in Adulthood (AOAH)) by assessing 170HP, DHEAS, cortisol, thyroid hormones, and prolactin levels (6).

Then based on the Rotterdam diagnostic criteria, two of the following three disorders are necessary:

1. Clinical Hyperandrogenism (Hirsutism) $(H)$.

Clinical Hyperandrogenism= The Ferriman-Gallwey score of 8 (15), and above or biochemical hyperandrogenism= elevated serum total testosterone levels or free androgen index (FAI)): hyperandrogenic serum total testosterone (TT), greater than 0.68 $\mathrm{ng} / \mathrm{ml}$ and free androgen index (FAl) More than 5.36\% (16). The TB and SHBG assay methods were measured by electro quantitative luminescence using a Roche German kit by Cobas E411. FAI= TT (nmol / I)/ SHBG (nmol / I)* 100 (17).

2. Ovulation disorder: (Oligo/anovulation)

The menstrual cycle of more than 35 days (oligomenorrhea) or more than 3 months (amenorrhea) (18).

3. Polycystic ovary view in ultrasound $(P)$

An ovarian volume greater than 10 cubic centimeters in at least one ovary or observation of more than 5 to 8 multiple fine follicles (18).

After entering the study, they were divided into the following four phenotypes:

1) Complete phenotype or $A(H+P+O)$

2) Ovulatory phenotype or $B(H+P)$

3) Normoandrogenic phenotype or C $(P+0)$

4) Phenotype or $\mathrm{D}(\mathrm{H}+\mathrm{O})(3)$.

Finally, 182 participants were included in the study (31 people in control group, 41 people in group A, 33 people in group B, 40 people in group $\mathrm{C}$, and 37 people in group $\mathrm{D}$ ). The researcher assessed physical activity by asking research units about whether or not to exercise. Participants' physical activity was measured at three levels based on their responses. Level 1: Normal daily activities without exercise, Level 2: Moderate physical activity: ( 1 to 2 times a week, each time for at least 20 minutes). High physical activity: (3 or more 3 times a week, Each time for at least 20 minutes) (19).

Anthropometric evaluations (height, weight, BMI), ovarian sonography, hirsutism as a clinical symptom of hyperandrogenism, hormonal tests were performed to determine serum androgens. Questions were raised about menstruation. Menstrual disorder was evaluated.

In this study, insulin resistance was assessed using HOMA index. The original HOMA model was described in 1985 with a formula for approximate estimation by Mathew et al. (1985) (20). The formula of the HOMA index is calculated by multiplying the fasting blood glucose concentration ( $\mathrm{mmol} / \mathrm{l}$ ) and the fasting insulin concentration (mmol / $\mathrm{ml}$ ) Divide by a fixed number of 22.5 (using fasting serum glucose and fasting insulin levels). The cut-off point for defining insulin resistance is considered based on the HOMA-IR criterion (cut off $>2.5)(21)$.

HOMA $-\mathrm{IR}=$ fasting glucose $(\mu \mathrm{U} / \mathrm{ml}) \times$ fasting insulin $(\mathrm{mmol} / \mathrm{l}) / 22.5$

$5 \mathrm{ml}$ of venous blood (To check fasting blood glucose, insulin levels, testosterone, and SHBG) were obtained from all research units in the laboratory and in the fasting state. Glucose was measured by glucose oxidase assay and insulin was measured by immunoradiometric method. Beck Man's immunotech kit was used to check the cases, with an extra-test accuracy of $3.4 \%$ 
and an intra-test accuracy of $4.3 \%$. Also in vitro measurement of SHBG and testosterone levels, the ELISA (Enzyme-Linked Immunosorbent Assay) method is IBL and DRG Kits, respectively.

SPSS software was used for data analysis and statistical tests. At first, Kolmogorov-Smirnov test was used for data normality. Parametric tests (for normal data) and non-parametric tests (for non-normal data) were used to analyze the data. Kruskal Wallis test (for non-normal data) and one-way ANOVA test (for normal data) were used to compare the variables. Spearman correlation test (because data were not normal) was used to investigate the relationship between quantitative variables. Chi-square test was also used to investigate some underlying variables. Significance level $\mathrm{P}<0.05$ was considered.

\section{Results}

The results of Kruskal Wallis, One-way ANOVA and Chi-square tests showed that there were no significant differences between the four subgroups of PCOS women and the control group in terms of age $(P=0.099)$, body mass index $(P=0.990)$, education level $(P=0.309)$, economic status $(P=0.275)$, and physical activity status $(P=0.871)$. The two groups were homogeneous $(p>0.05)$. (Table 1$)$.

Table 1

Comparison of demographic components at baseline

\begin{tabular}{|c|c|c|c|c|c|c|}
\hline $\begin{array}{l}\text { Demographic } \\
\text { Components }\end{array}$ & $\begin{array}{l}\text { Phenotype } \\
\text { A: } \mathrm{H}+0+ \\
\text { P } \\
\mathrm{N}=41\end{array}$ & $\begin{array}{l}\text { Phenotype B: } \mathrm{H}+ \\
\text { P } \\
N=33\end{array}$ & $\begin{array}{l}\text { Phenotype } \\
\text { C: } 0+P \\
N=40\end{array}$ & $\begin{array}{l}\text { Phenotype } \\
\text { D: } \mathrm{H}+0 \\
N=37\end{array}$ & $\begin{array}{l}\text { Control } \\
\text { groupe } \\
\mathrm{N}=31\end{array}$ & P -value \\
\hline Age & $\begin{array}{l}28.07 \pm \\
4.70\end{array}$ & $27.00 \pm 5.44$ & $\begin{array}{l}29.70 \pm \\
6.44\end{array}$ & $\begin{array}{l}29.83 \pm \\
5.93\end{array}$ & $28.07 \pm 4.70$ & $\begin{array}{l}0.099 \\
\text { K.W }\end{array}$ \\
\hline BMI & $\begin{array}{l}25.48 \pm \\
5.23\end{array}$ & $25.06 \pm 4.28$ & $\begin{array}{l}25.08 \pm \\
3.93\end{array}$ & $\begin{array}{l}24.98 \pm \\
4.80\end{array}$ & $25.48 \pm 5.23$ & $\begin{array}{l}0.990 \\
\text { K.W }\end{array}$ \\
\hline \multicolumn{7}{|l|}{ Educational status } \\
\hline Under the Diploma & $4(9.8)$ & $3(9.1)$ & $4(10.0)$ & $2(5.4)$ & $2(6.5)$ & \multirow{4}{*}{$\begin{array}{l}0.309 \\
\text { Chi- } \\
\text { square }\end{array}$} \\
\hline $\begin{array}{l}\text { Graduate Diploma/ } \\
\text { Diploma }\end{array}$ & $9(22.0)$ & $5(15.2)$ & $10(25.0)$ & $15(43.2)$ & $14(45.2)$ & \\
\hline Bachelor & $19(46.3)$ & $16(48.5)$ & $20(50.0)$ & $16(40.5)$ & $10(32.3)$ & \\
\hline $\begin{array}{l}\text { Masters degree and } \\
\text { higher }\end{array}$ & $9(22.0)$ & $9(27.3)$ & $6(15.0)$ & $4(10.8)$ & $5(16.1)$ & \\
\hline \multicolumn{7}{|c|}{ The economic situation } \\
\hline Poor & $16(39.0)$ & $23(69.7)$ & $23(57.5)$ & $21(56.8)$ & $17(54.8)$ & \multirow{3}{*}{$\begin{array}{l}0.275 \\
\text { Chi- } \\
\text { square }\end{array}$} \\
\hline medium & $16(39.0)$ & $5(15.2)$ & $11(27.5)$ & $7(18.9)$ & $7(22.6)$ & \\
\hline Good & $9(22.0)$ & $5(15.2)$ & $6(15.0)$ & $9(24.3)$ & $7(22.6)$ & \\
\hline \multicolumn{7}{|c|}{ Physical activity status } \\
\hline Level 1 & $23(56.1)$ & $20(60.6)$ & $25(62.5)$ & $25(67.6)$ & $20(67.6)$ & 0.871 \\
\hline Level 2 & $6(14.6)$ & $6(18.2)$ & $7(17.5)$ & $5(13.5)$ & $5(13.5)$ & \multirow{2}{*}{$\begin{array}{l}\text { Chi- } \\
\text { square }\end{array}$} \\
\hline Level 3 & $12(29.3)$ & $7(21.2)$ & $8(20.0)$ & $7(18.9)$ & $6(18.9)$ & \\
\hline
\end{tabular}


Insulin resistance status was compared with the HOMA-IR index (Cut off $>2.5$ ) in PCOS and control groups with the Chisquare test. There was a significant difference in the index of insulin. The number of women with polycystic ovary syndrome who also had insulin resistance was higher in phenotype A (complete PCOS phenotype (no ovulation, hirsutism, and sonographic abnormality)) than in other groups (61\%).

In phenotype B (ovulation phenotype (hirsutism and sonographic disorder without ovulation disorder), $51.5 \%$, phenotype $\mathrm{C}$ (ovulation and hirsutism disorder), 37.5\% and better than the other groups, in phenotype D ((Ultrasound view impairment and no ovulation), $48.6 \%$ had PCOS and HOMA-IR at the same time, In the control group, although they had no symptoms of PCOS, two (6.5\%) patients had the syndrome. (Table 2).

Table 2

Comparison of Insulin Resistance Status with HOMA-IR Index in PCOS Subgroups and Control group

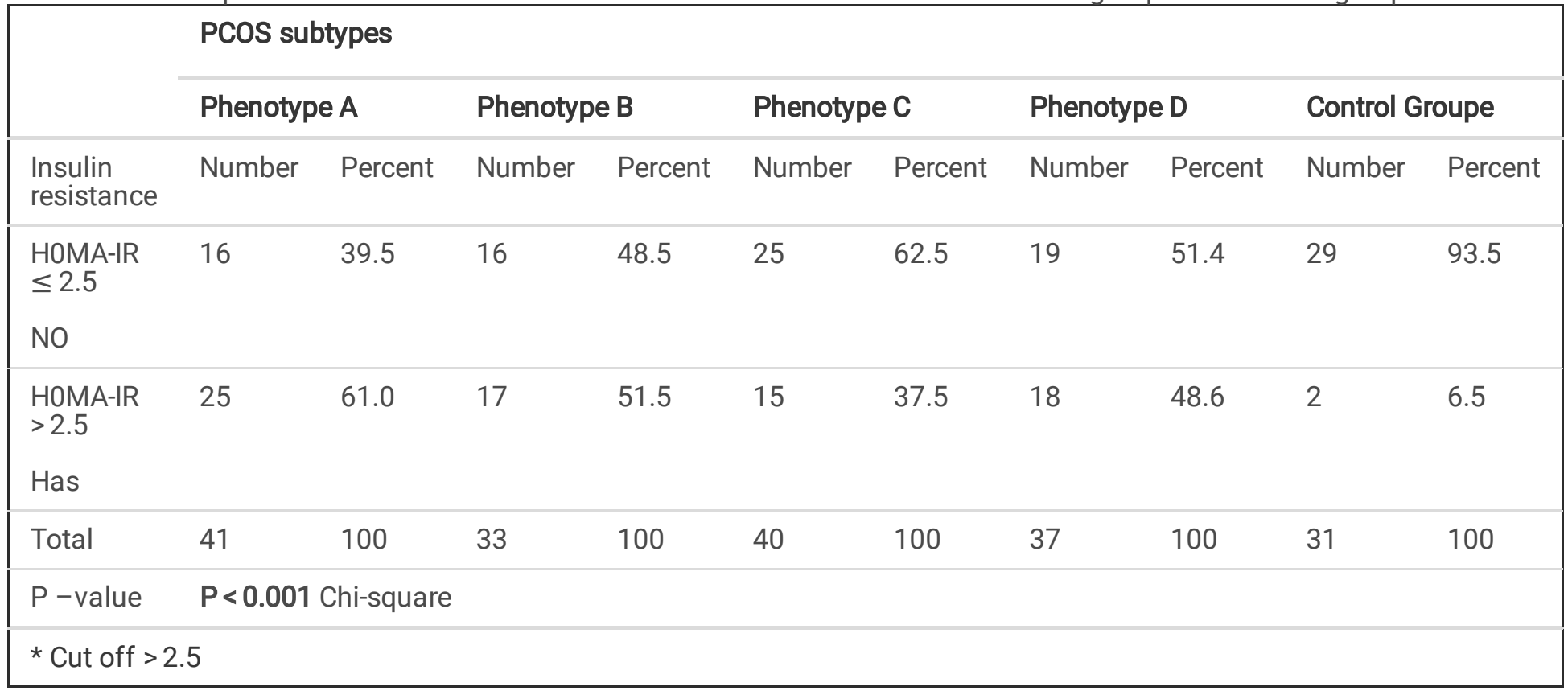

In the study of serum androgens, mean TT and FAI were lower in the control group than in patients with PCOS. Mean of TT and FAI among PCOS subgroups were as follows $\mathrm{C}<\mathrm{B}<\mathrm{D}<\mathrm{A}$. Comparison between groups with chi-square test showed a significant difference between FAI and TT in PCOS subgroups and control group $(P<0.001)$.

Mean SHBG was highest in the control group and lowest in the D phenotype.A comparison between groups with chi-square test showed that the mean SHBG was significantly different between PCOS and control groups $(P<0.001)$. (Table 3). 
Table 3

Comparison of mean androgenic component differences In PCOS subtype and control group

\begin{tabular}{|lllll|}
\hline Studied groups & FAl & SHBG(ng/ml) & TT(ng/ml) \\
\cline { 2 - 5 } & & Mean \pm SD & Mean \pm SD & Mean \pm SD \\
\hline Case Group (PCOS Subgroups) & Phenotype A & $8.92 \pm 7.75$ & $41.53 \pm 27.23$ & $0.77 \pm 0.59$ \\
\cline { 2 - 5 } & Phenotype B & $4.05 \pm 4.52$ & $51.16 \pm 28.31$ & $0.49 \pm 0.42$ \\
\cline { 2 - 5 } & Phenotype C & $3.17 \pm 2.93$ & $50.50 \pm 23.03$ & $0.33 \pm 0.14$ \\
\cline { 2 - 5 } & Phenotype D & $5.51 \pm 3.62$ & $39.81 \pm 20.27$ & $0.55 \pm 0.40$ \\
\hline Control Group & & $1.45 \pm 1.51$ & $76.32 \pm 45.10$ & $0.22 \pm 0.16$ \\
\hline Test Kruskal Wallis & P-value & P<0.001 & P $<0.001$ & P $<0.001$ \\
\hline H: Clinical +/ Paraclinical Hyperandrogenism O: Oliogo/Anovulation P: Pco sonographic view & & & \\
\hline A: $\mathrm{H}+\mathrm{O}+\mathrm{P}$ & & & & \\
\hline B: $\mathrm{H}+\mathrm{P}$ & & & & \\
\hline C: $\mathrm{O}+\mathrm{P}$ & & & & \\
\hline D: $\mathrm{H}+\mathrm{O}$ & & & & \\
\hline
\end{tabular}

Spearman test showed a significant relationship between the insulin resistance index (HOMA-IR) and serum androgen levels (SHBG, FAI). Subgroups were as follows: In phenotype A, there was a significant positive relationship between HOMA-IR and FAI $(P=0.014)$ and there was a significant negative relationship between HOMA-IR and SHBG $(P<0.001)$. In phenotype $C$, there was a significant negative relationship between HOMA-IR and SHBG $(P=0.002)$. In phenotype $D$, there was a significant positive relationship between HOMA-IR and FAI $(P=0.035)$. In phenotype $B$ and control groups, there was no relationship between HOMA-IR and serum androgens levels $(P>0.05)$. Also, in all the different PCOS phenotypes, there was no significant relationship between HOMA-IR and serum TT level $(P>0.05)$. (Table 4). 
Table 4

Evaluation of correlation between HOMA insulin resistance index and androgenic components in four pcos subgroups and control group

\section{Evaluation of correlation between Androgenic components and HOMA insulin resistance index in subgroup A $(\mathrm{H}+\mathrm{O}+\mathrm{P})$}

\begin{tabular}{|lllll|}
\hline Androgenic components & & TT & SHBG & FAI \\
\hline HOMA-IR & R & 0.382 & -0.595 & 0.053 \\
\cline { 2 - 4 } & $\mathrm{P}$ & 0.740 & $<0.001$ & $\mathbf{0 . 0 1 4}$
\end{tabular}

Evaluation of correlation between Androgenic components and HOMA insulin resistance index in subgroup B $(\mathrm{H}+\mathrm{P})$

$\begin{array}{lllll}\text { Androgenic components } & & \text { TT } & \text { SHBG } & \text { FAI } \\ \text { HOMA-IR } & \mathrm{R} & -0.023 & -0.185 & 0.068 \\ & \mathrm{P} & 0.900 & 0.303 & 0.707\end{array}$

Evaluation of correlation between Androgenic components and HOMA insulin resistance index in subgroup $\mathrm{C}(\mathrm{P}+\mathbf{0})$

$\begin{array}{lllll}\text { Androgenic components } & & \text { TT } & \text { SHBG } & \text { FAI } \\ \text { HOMA-IR } & \text { R } & 0.257 & -0.470 & 0.150 \\ & \text { P } & 0.110 & \mathbf{0 . 0 0 2} & 0.356\end{array}$

Evaluation of correlation between Androgenic components and HOMA insulin resistance index in subgroup D $(H+0)$

\begin{tabular}{lllll} 
Androgenic components & & TT & SHBG & FAl \\
HOMA-IR & $\mathrm{R}$ & 0.201 & -0.217 & 0.347 \\
\cline { 2 - 4 } & $\mathrm{P}$ & 0.234 & 0.198 & $\mathbf{0 . 0 3 5}$
\end{tabular}

Evaluation of correlation between Androgenic components and HOMA insulin resistance index in Control group

\begin{tabular}{lllll} 
Androgenic components & & TT & SHBG & FAl \\
HOMA-IR & $\mathrm{R}$ & 0.259 & -0.330 & -0.104 \\
\cline { 2 - 4 } & $\mathrm{P}$ & 0.159 & 0.070 & 0.579
\end{tabular}

\section{Discussion}

This study showed that insulin resistance index (HOMA-IR) in different PCOS phenotypes correlated with serum androgen levels (SHBG, FAI). In phenotype A, there was a positive relationship between HOMA-IR and FAI and there was a negative relationship between HOMA-IR and SHBG. In phenotype C, there was a negative relationship between HOMA-IR and SHBG. In phenotype $D$, there was a positive relationship between HOMA-IR and FAl. In phenotype B and control groups, there was no relationship between HOMA-IR and serum androgens levels. Also, in all the different PCOS phenotypes, there was no relationship between HOMA-IR and serum TT levels.

Tsai et al.'s (2013) study was in line with the present study. This study was performed on 45 women with PCOS and 161 infertile and non-PCOS women with the aim of comparing glucose metabolism and sex hormones. Results showed that SHBG had a negative association with HOMA-IR and FAI in women with non-PCOS And SHBG have a positive association with HOMA-IR in women with non-PCOS (22). In Jayagopal's (2003) study, SHBG was negatively correlated with HOMA-IR in women with PCOS and it was suggested that SHBG should be used as a predictor of IR in these women (23). In the study of Kajaia (2007), women classified using insulin resistance indices showed significantly higher BMI and FAl and lower levels of SHBG and HDL. However, SHBG may act as a predictive marker of IR in these women, especially in those who are obese (13). 
In Ducluzeau's (2003) study, sex hormone binding globulin (SHBG) was associated with IR. But SHBG cannot act as an IR predictor. In this study, both obese and lean women were exposed to insulin resistance (8).

Osuna's (2006) study was inconsistent with the present study. The purpose of this study was to investigate the relationship of steroid hormones with HOME-IR in 77 men aged 20-60 years. The study units were categorized into three groups with different BMIs. The results showed that TT and SHBG values were related to HOME-IR. In the present study, TT was not associated with HOME-IR (24). Perhaps the reason for this discrepancy is the difference in research units. In the Osuna study, all were men, and men had higher TT levels than women. Osuna's study measured different BMls of resistance index, but in the present study, individuals were homogeneous in BMI.

In PCOS, insulin-independent of gonadotropin secretion induces steroidogenesis in the ovary. Insulin has direct and indirect roles in PCOS hyperandrogenism. Insulin, in combination with LH, increases androgen production in Teca cells. Insulin also inhibits the liver's production of sex hormone-bound globulin, thereby increasing the ratio of available testosterone. On the other hand, overproduction of androgens and hyperandrogenism and hyperphosphorylation of the $\beta$-insulin receptor leads to inhibition of tyrosine phosphorylation of the insulin receptor-beta. Inhibition of the insulin beta receptor leads to insulin resistance in women with PCOS. Under normal circumstances, activation of the insulin beta-receptor requires tyrosine phosphorylation of the amino acid, which induces an insulin response at the receptor surface. In conditions of hyperandrogenism, serine beta-receptor hyperphosphorylation prevents tyrosine amino acid phosphorylation (25).

Based on the findings of the present study, increased insulin resistance leads to an increase in the FAl profile and a decrease in SHBG in A subgroup. Increased insulin resistance leads to a decrease in SHBG in C subgroup and an increase in the FAI profile in the $D$ subgroup, and consequently impaired hormonal profile exacerbates insulin resistance. Findings of the present study showed no association between insulin resistance and serum androgens levels. People with PCOS who are subgroup B have regular menstrual cycles, which may indicate menstrual cycles with ovulation. In this subgroup, the mean HOMA-IR was lower than other PCOS phenotypes and among the high androgen, subgroups had the lowest levels of testosterone and FAl, which may possibly justify this lack.

In the study of serum androgens levels, mean TT and FAI were lower in the control group than in the patients with PCOS, and in PCOS subgroups were A > D > B > C. In comparison between PCOS subgroups in terms of androgenic factors, Subgroup C exhibits the mildest condition of PCOS compared to other PCOS subtypes in the absence of symptoms of hyperandrogenism (clinical signs of hyperandrogenism or in vitro increase of androgenic components).

Among the other three subgroups of PCOS that exhibited hyperandrogenemia, Subgroup A had the highest and Subgroup B had the lowest levels of FAl and total testosterone. In most of the studies studied, the highest level of androgenic components was in subgroup A, which could indicate deterioration of condition of this subgroup compared to the other two subgroups. Women with PCOS who are subgroup B have regular menstrual cycles, which may indicate menstrual cycles with ovulation, which may indicate lower testosterone and FAl levels in this subgroup than in high androgen subgroups (A and D).

The study by Guastella et al. (2010) is in line with the present study. This study was conducted in Palermo, Italy on 382 women with PCOS and 85 healthy women in the control group. The $A(O+H+P)$ and $D(O+H)$ phenotypes had significantly higher TT and FAl levels than the control group and the $B(H+P)$ and $(O+P) C$ phenotypes $(26)$. The results of this study are consistent with the present study.

But the study by Yilmaz et al. (2011) shows different results. The Yilmaz study was performed on 127 women with PCOS based on Rotterdam diagnostic criteria and 44 women in the control group. The mean TT and FAI were not significantly different among the three subgroups with hyperandrogenism (27). In this study, the number of people in each subgroup was significantly different from each other, which may influence their results.

In the present study, the insulin resistance index with the HOMA-IR index was the highest in phenotype $A$ and in phenotype $C$ was better than others. The number of women with polycystic ovary syndrome who also had insulin resistance was lower 
than the other groups. Many studies have reported that PCOS in subgroup A (classical form) is more insulin resistant than subgroup B (ovulatory form) $(28,29)$, and subgroup C (normal androgenic) $(29,30)$. The study of HY Zhang et al $(2009)$ in China is in line with the results of the present study. The results of this study showed that the concentration of TT, IR, and F-G index in the subgroup $A(O+H+P)$ was the highest and in the subgroup $(O+P) C$ and in the control group was the lowest (31). In the study of Tehrani et al (2014), phenotypes $A(O+H+P)$ and $B(H+P)$ had the highest levels of insulin and HOMA-IR compared to other phenotypes (15). In the Yilmaz study, HOMA index was highest in $(\mathrm{H}+\mathrm{O})$ and $(\mathrm{P}+\mathrm{H}+\mathrm{O})$ phenotypes and was lowest in $(\mathrm{H}+\mathrm{P})$ and $(\mathrm{P}+\mathrm{O})$ phenotypes. The prevalence of insulin resistance in the phenotype $(\mathrm{P}+\mathrm{O})$ was closer to the control group than other phenotypes (29). These results are in line with the present study.

Some studies report different results than ours. Zhang et al. (2012) conducted a case-control study on 406 PCOS patients and 342 women in the control group, between 17 and 40 years from a population in southwest China between 2006 and 2011. PCOS diagnosis was based on the modified Rotterdam criteria. The results reported that the two-hour insulin and glucose levels in the subgroup $(\mathrm{H}+\mathrm{O}+\mathrm{P})$ were higher than the other three subtypes, but the HOMA-IR index was not significantly different between the four subtypes (32). Perhaps the reason for the difference between the results and the present study is the different number and ethnicity of the research units.

In the 2007 study, Shroff et al performed on 258 women with PCOS (Rotterdam diagnostic criteria) and 110 healthy women in the control group. Insulin resistance was calculated using the HOMA-IR and QUICKI indices. There was no significant difference in the prevalence of insulin resistance between the subtypes (33).

Cross-sectional study of Kar et al. 2013 in India performed on 269 patients with phenotype $(\mathrm{P}+\mathrm{H}+0), 91$ patients with phenotype $(P+O), 46$ patients with phenotype $(H+O), 4$ patients with phenotype $(P+H)$ done. The results showed that HOMA-IR index was not significantly different between the subgroups (3).

The Panidis et al. (2011) study performed on 1212 women with PCOS and 254 healthy women (control group) matched for BMI. Both normal and obese and overweight and obese women had a significant difference in insulin resistance compared to the control group. Obese or overweight women of phenotype $D$ had higher insulin resistance than the control group, whereas insulin resistance in phenotype $\mathrm{C}$ was not significantly different from the control group. Circulating androgens were more in women with phenotype one to three compared to phenotype D and in phenotype A more than phenotype B and C. Insulin resistance was assessed using HOMA-IR and QUICKI indices and no significant differences were observed between the subgroups (34).

\section{Conclusions}

Insulin resistance is one of the major components of PCOS and an introduction to diseases such as metabolic syndrome, type 2 diabetes and cardiovascular disease. Consequently, because of the interaction between insulin resistance and some of the endogenous hormone components in PCOS, it is recommended to evaluate PCOS patients for insulin resistance, especially in subgroups with elevated serum androgen levels. It is hoped that measures will be taken early in identifying atrisk patients through primary biochemical markers to prevent and change lifestyle. Therefore, it is suggested that clinical trials based on the effect of lifestyle changes (weight, sleep, exercise, nutrition, stress) on insulin resistance and serum androgens levels in women with polycystic ovary syndrome be considered.

\section{Abbreviations}

PCOS (Polycystic Ovary Syndrome)

ESHRE (European Society of Human Reproduction and Embryology)

HOMA (Homeostasis Model Assessment Method)

HOMA-IR (Insulin Resistance calculated by the Homeostasis Model Assessment Method)

Page $10 / 13$ 
F-G (Ferrimen-Gallwey score)

FAl (Free Androgen Index)

SHBG (Sex hormone-binding globulin)

TT (Total Testosterone)

HA (Hyperandrogenism).

\section{Declarations}

Research limitations: The limitations of this study include the lack of cooperation of many physicians in requeste.

Ethics approval and consent to participate: This study was conducted with the approval of the Institutional Review Board (IRB), and of Tarbiat Modares University's Ethics Committee (IR.REC.1395. D52/5503).

Consent for publication: All the women were informed about the aims of the project and gave written consent before participating in the study.

Availability of data and materials: The datasets used and/or analyzed during the current study are available from the corresponding author on reasonable request.

Competing interests: The authors declare that they have no competing interests

Funding: The authors declare that they have no funding.

Authors' contributions: Movahedinejad Maryam, Kamali Zahra , Ziaei Saeide study concept and design; Movahedinejad Maryam acquisition of data; Kazemnejad Anooshiravan, Kamali Zahra, analysis and interpretation of data; Ziaei Saeide, Movahedinejad Maryam, , Kamali Zahra drafting of the manuscript; Movahedinejad Maryam, Kamali Zahra, critical revision of the manuscript for important intellectual content; Kamali Zahra, Kazemnejad Anooshiravan statistical analysis; Kamali Zahra, Ziaei Saeide, administrative, technical, and material support. All authors read and approved the final manuscript.

Acknowledgements: In this regard, we thank the Vice-Chancellor for Research in Tarbiat Modares University of Medical Science, Tehran, Iran. It should be noted that the respected personnel of the women's ward of Arash Hospital in Tehran is thanked for their cooperation in the research.

Conflict of interest: There were no conflicts of interest in this study.

\section{References}

1. Norman RJ DD, Legro RS, Hickey TE. Polycystic ovary syndrome. Lancet. 2007; 370:685-697.

2. Ding T HP, Petersen I, Wang FF, Qu F, Baio G. The prevalence of polycystic ovary syndrome in reproductive-aged women of different ethnicity: a systematic review and meta-analysis. Oncotarget. 2017 Nov 10;8(56):96351.

3. Kar S. Anthropometric c, and metabolic comparisons of the four Rotterdam PCOS phenotypes: A prospective study of PCOS women. Journal of human reproductive sciences. 2013;6(3):194-200.

4. Mortensen M ED, Little JE. Asymptomatic volunteers with a polycystic ovary syndrome are functionally distinct but heterogenous population. J Clin Endocrinol Metab 2009; 94:1579-86.

5. Azziz R, Carmina E, Dewailly D, Diamanti-Kandarakis E, Escobar-Morreale HF, Futterweit W, et al. The Androgen Excess and PCOS Society criteria for the polycystic ovary syndrome: the complete task force report. Fertility and sterility.

Page $11 / 13$ 
2009;91(2):456-88.

6. Berek.novak J. Berek and Novak s gynecology-15th ed. 2012.

7. Ehrmann DA. Hirsutism and Virilization. In: Longo DL KD, Jameson JL, Fausi AS, Hauser SL, Loscalzo J. Harrison's principles of internal medicine. 18th ed. New York: McGraw Hill; 2012. p. 380-2.

8. Ducluzeau PH CP, Malvoisin E, Bornet H, Vidal H, Laville M, Pugeat M. Glucose-to-insulin ratio rather than sex hormonebinding globulin and adiponectin levels is the best predictor of insulin resistance in nonobese women with polycystic ovary syndrome. The Journal of Clinical Endocrinology \& Metabolism. 2003 Aug 1;88(8):3626-31.

9. Marshall JC, Dunaif A. All women with PCOS should be treated for insulin resistance. Fertil. Steril. 2012;97(1):18-22

10. Rosenfield RL. Polycystic ovary syndrome and insulin-resistant hyperinsulinemia. Journal of the American academy of Dermatology. 2001;45(3):S95-S104.

11. Zaimzadeh N ZS, Mohammadzadeh N, Alizadeh Otaghvar H, Mottaghi A. Comparison of vitamin D dietary intake among four phenotypes of polycystic ovary syndrome and its association with serum androgenic components. Razi Journal of Medical Sciences. 2018 May 15;25(167):87-96.

12. Rosenfield RL BR, Cara JF, Lucky AW. Dysregulation of cytochrome P450c 17 alpha as the cause of polycystic ovarian syndrome. Fertil Steril 1990; 53:785-791.

13. Kajaia N BH, Dittrich R, Oppelt PG, Flor B, Cupisti S, Beckmann MW, Mueller A. Low sex hormone-binding globulin as a predictive marker for insulin resistance in women with hyperandrogenic syndrome. Eur J Endocrinol. 2007;157:499-507.

14. Traub ML. Assessing and treating insulin resistance in women with polycystic ovarian syndrome. World journal of diabetes. 2011;2(3):33.

15. Tehrani FR RH, Khomami MB, Tohidi M, Azizi F. The prevalence of metabolic disorders in various phenotypes of polycystic ovary syndrome: a community based study in Southwest of Iran. Reprod Biol Endocrinol. 2014;12(89):10.1186.

16. Hashemi S TF, Noroozzadeh M, Azizi F. Normal cut-off values for hyperandrogenaemia in Iranian women of reproductive age. European Journal of Obstetrics \& Gynecology and Reproductive Biology. 2014;172:51-5.

17. Goodarzi MO DD, Chazenbalk G, Azziz R. Polycystic ovary syndrome: etiology, pathogenesis and diagnosis. Nature Reviews Endocrinology. 2011;7(4):219-31.

18. Schorge JO HB, Bradshaw KD, Halvorson LM, Schaffer JI, Corton MM. Williams gynecology: McGraw-Hill Medical New York; 2008.

19. Kim HM, Park J, Ryu SY, Kim J. The effect of menopause on the metabolic syndrome among Korean women: the Korean National Health and Nutrition Examination Survey, 2001. Diabetes care. 2007;30(3):701-6.

20. Matthews DR HJ, Rudenski AS, Naylor BA, Treacher DF, Turner RC. Homeostasis model assessment: insulin resistance and $\beta$-cell function from fasting plasma glucose and insulin concentrations in man. Diabetologia. 1985 Jul 1;28(7):412-9.

21. Wallace TM LJ, Matthews DR. Use and abuse of HOMA modeling. Diabetes care. 2004; 27(6): 1487- 95.

22. Tsai Y-H, Wang T-W, Wei H-J, Hsu C-Y, Ho H-J, Chen W-H, et al. Dietary intake, glucose metabolism and sex hormones in women with polycystic ovary syndrome (PCOS) compared with women with non-PCOS-related infertility. British journal of nutrition. 2013;109(12):2190-8. 
23. Jayagopal V, Kilpatrick E, Jennings P, Hepburn D, Atkin S. The biological variation of testosterone and sex hormonebinding globulin (SHBG) in polycystic ovarian syndrome: implications for SHBG as a surrogate marker of insulin resistance. The Journal of Clinical Endocrinology \& Metabolism. 2003;88(4):1528-33.

24. Osuna C J, Gomez-Perez R, Arata-Bellabarba G, Villaroel V. Relationship between BMI, total testosterone, sex hormonebinding-globulin, leptin, insulin and insulin resistance in obese men. Archives of andrology. 2006;52(5):355-61.

25. Sung Y-A, Oh J-Y, Chung H, Lee H. Hyperandrogenemia is implicated in both the metabolic and reproductive morbidities of polycystic ovary syndrome. Fertility and sterility. 2014;101(3):840-5.

26. Guastella E LR, Carmina E. Clinical and endocrine characteristics of the main polycystic ovary syndrome phenotypes. Fertility and sterility. 2010; 94(6): 2197- 201.

27. Yilmaz M IU, Delibas IB, Kadanali S. Anthropometric, clinical and laboratory comparison of four phenotypes of polycystic ovary syndrome based on Rotterdam criteria. Journal of Obstetrics and Gynaecology Research. 2011;37(8):1020-6.

28. Carmina E CM, Longo RA, Rini GB, Lobo RA. Phenotypic variation in hyperandrogenic women influences the findings of abnormal metabolic and cardiovascular risk parameters. J Clin Endocrinol Metab; 2005 .90(5):2545-9.

29. Yilmaz M, Isaoglu U, Delibas IB, Kadanali S. Anthropometric, clinical and laboratory comparison of four phenotypes of polycystic ovary syndrome based on Rotterdam criteria. Journal of Obstetrics and Gynaecology Research. 2011;37(8):1020-6.

30. Dewailly D, Catteau-Jonard S, Reyss A-C, Leroy M, Pigny P. Oligoanovulation with polycystic ovaries but not overt hyperandrogenism. The Journal of Clinical Endocrinology \& Metabolism. 2006;91(10):3922-7.

31. Zhang HY ZF, Xiong J, Shi XB, Fu SX. Characteristics of different phenotypes of polycystic ovary syndrome based on the Rotterdam criteria in a large-scale Chinese population. BJOG : an international journal of obstetrics and gynaecology. 2009;116(12):1633-9.

32. Zhang J FP, Liu H, Bai H, Wang Y, Zhang F. Apolipoprotein Al and B levels, dyslipidemia and metabolic syndrome in south-west Chinese women with PCOS. Human reproduction. 2012;27(8):2484-93.

33. Shroff R SC, Davis W, Van Voorhis BJ, Dokras A. Risk of metabolic complications in the new PCOS phenotypes based on the Rotterdam criteria. Fertility and sterility. 2007;88(5):1389-95.

34. Panidis D TK, Misichronis G, Papadakis E, Betsas G, Katsikis I, et al. Insulin resistance and endocrine characteristics of the different phenotypes of polycystic ovary syndrome: a prospective study. Human reproduction. 2011:der418. 